\title{
Characterization of Fusarium solani (Mart.) Sacc. Causing Fusarium wilt of Bitter gourd in Coimbatore Region
}

\author{
J. Manohari $^{1 *}$, P. Latha ${ }^{1}$, A. Kamalakannan ${ }^{1}$, S. Selvakumar ${ }^{2}$ and M. Karthikeyan ${ }^{1}$ \\ ${ }^{1}$ Department of Plant Pathology, ${ }^{2}$ Department of Soil and \\ Water Conservation, Tamil Nadu Agricultural University \\ Coimbatore - 641 003, India \\ *Corresponding author
}

\section{A B S T R A C T}

\begin{tabular}{|l|}
\hline Ke y w o r d s \\
$\begin{array}{l}\text { Fusarium } \text { wilt, } \\
\text { Bitter gourd, } \\
\text { Pathogenicity, } \\
\text { Morphological and } \\
\text { molecular } \\
\text { characterization }\end{array}$ \\
\hline Article Info \\
\hline $\begin{array}{l}\text { Accepted: } \\
18 \text { May } 2020 \\
\text { Available Online: } \\
\text { 10 June } 2020\end{array}$ \\
\hline
\end{tabular}

Fusarium wilt of bitter gourd is caused by Fusarium spp. Ten isolates of Fusarium were isolated from bitter gourd growing areas of Coimbatore district to study the cultural and morphological characters of Fusarium sp. From the survey, it was found that the highest PDI was recorded in Naatukalpalayam 1 with $52.63 \%$. The mycelial diameter of ten isolates was ranged from 6.2 to $9.0 \mathrm{~cm}$ at seven days of incubation on PDA. The highest colony diameter of $9.0 \mathrm{~cm}$ was observed in $\mathrm{BF} 2$ and $\mathrm{BF} 7$ followed by BF5, BF8, BF9, BF4, BF6, BF10 and BF3. Minimum colony diameter was observed in BF1 with $6.1 \mathrm{~cm}$. Different media studies have shown that Fusarium solani could grow well in PDA and PSA. All ten isolates have produced micro, macroconidia and chlamydospores. The size $(\mu \mathrm{m})$ of microconidia ranged from (9.211.8) $\mathrm{x}(3.2-4.6)$ and macroconidia is (15.7-24.4) $\mathrm{x}(3.8-5.0)$ and they are hyaline in color. There are 2-4 septations in macroconidia. Macroconidia are sickle shaped with pointed tips and microconidia are elongated oval in shape. Chlamydospores were seen on intercalary, terminal portion of mycelium and oval in shape. PCR amplification with universal primers of ITS1 and ITS4 yielded approximately at $560 \mathrm{bp}$. Sequencing of ITS regions followed by BLAST search confirmed the associated fungus as Fusarium solani.

\section{Introduction}

Bitter gourd (Momordica charantia L.) is one of the important vegetables which belongs to cucurbit members. In India, bitter gourd has positioned a prominent place because of its year-round cultivation and export potential. The crop is cultivated over an area of 1492 ha in Tamil Nadu with an average production of 30,520 tonnes and the productivity of 20.25 tha $^{-1}$. The major problem in bitter gourd production is Fusarium wilt and it is considered to be the most destructive soil borne disease which is responsible for yield reduction (Tamilselvi, 2016). This disease is found to cause economic loss ranging from 30 
to 50 percent during dry warm conditions (Tamilselvi and Pugalendhi, 2015). In general, Fusarium wilt is caused by Fusarium oxysporum and served as a major reason for great loss on some economically important cucurbit crops like watermelon, cucumber. In bitter gourd, this Fusarium wilt was found to be caused by the pathogen Fusarium solani (Sreegayathri et al., 2018). The major symptoms of Fusarium wilt are damping off, seedling disease and wilting at any developmental stage of the crop. On matured plants the symptoms appeared as dull grey green leaves followed by yellowing of foliage, wilting during day hours and eventually leads to death. The characterisation of pathogen is important to decide on the plant protection measure and hence in this study, Fusarium spp. which causes Fusarium wilt in bitter gourd were isolated from different regions of Coimbatore, and their cultural and morphological characters were studied and the molecular characterisation was done. This will be helpful for the further studies related to Fusarium wilt of bitter gourd.

\section{Materials and Methods}

\section{Survey and isolation of Fusarium spp.}

A random fieldsurvey was conducted in the bitter gourd cultivating areas of Coimbatore district, percent disease incidence was calculated $(\mathrm{PDI}=$ No. of infected plants $\mathrm{x} 100$ / Total no. of plant assessed) and symptoms were collected to isolate the pathogen. The typical symptom of vascular discoloration was observed. The discolored portions were cut into small pieces and surface sterilized with $70 \%$ ethanol and then washed well with sterile water for three times. All these steps have done under the aseptic condition. For isolation of pathogen, Potato Dextrose Agar (PDA) medium was used. The PDA medium was added with Cefotaxime antibiotic to prevent the bacterial contamination. The infected bits were placed in sterile petri dishes containing PDA medium and incubated at laboratory condition $\left(28 \pm 2^{\circ} \mathrm{C}\right)$. After five days of incubation, pure culture of Fusarium was obtained by single spore isolation technique and maintained in PDA slant at $5^{\circ} \mathrm{C}$ for further study.

\section{Pathogenicity}

The pathogenicity of Fusarium was proved by the soil inoculation method. The virulent isolate BF2 was multiplied in the sand maize meal containing sand and ground maize at the ratio of 9:1. The mixture was slightly moistened with water and sterilized at $121^{\circ} \mathrm{C}$ at 15 psi for 2 hours. The fungus was inoculated into autoclaved medium and incubated for 15 days at room temperature for multiplication. Bitter gourd seeds (variety: Co 1) were raised in propagation tray. Potting soil (red soil : sand : cow dung manure @ 1: 1: 1 ratio) was sterilized in autoclave for 2 consecutive days. The inoculum on sand maize medium was incorporated into sterilized soil at the rate of $50 \mathrm{~g} \mathrm{~kg}^{-1}$ of the soil. Twenty-five days old seedlings were transplanted into the pots and maintained under glasshouse conditions. Three replications were maintained and monitored regularly. Control plant was maintained without inoculating the pathogen. From the infected plants, pathogen was reisolated and Koch's postulates were proved.

\section{Cultural and morphological characterization of pathogen}

Ten isolates of Fusarium were grown on PDA for studying their cultural characters. Tenmillimeter mycelial disc of the fungus from actively growing culture plates were taken and placed on PDA media contained in $(90 \mathrm{~mm})$ sterile petri dishes. They were incubated at room temperature for 7 days and 
observations were recorded on parameters like colony diameter, colony character, pigmentation and colony margin. The colony diameter $(\mathrm{cm})$ was measured at 48, 72, 96, 144 and 168 hours after incubation. The experiment was laid out in completely randomized design with three replications. The colony diameter was measured from all the four sides.

Spores of all the ten Fusarium isolates were collected. Spores were observed under high power objectives $(40 \mathrm{X})$ and their images were captured. The average size of the spores was measured and shape of the spores were also recorded. For micro and macroconidia, length $(\mu \mathrm{m})$ and breadth $(\mu \mathrm{m})$ of twenty-five (25) spores for all the isolates were measured from fifteen days old culture.

\section{Molecular characterization}

DNA were extracted for all the isolates of Fusarium and PCR amplification was performed with universal primers ITS 1 ( $5^{\prime}-$ CTTGGTCATTTAGAGGAAGTAA - 3') and ITS $4\left(5^{\prime}-\right.$ TCCTCCGTTATTGA TATGC -3 '). PCR reactions were performed in $10 \mu \mathrm{l}$ final volume containing $5 \mu 1$ of Taq $2 x$ master mix (Ampliqon) which contains 1.5 $\mathrm{Mm} \mathrm{MgCl}_{2}, 2 \mu \mathrm{l}$ double distilled water, $1 \mu \mathrm{l}$ of each primer, and $1 \mu \mathrm{l}$ of DNA. Optimal PCR efficacy was obtained with an initial denaturation of $95^{\circ} \mathrm{C}$ for $3 \mathrm{~min}$ followed by 35 amplification cycles (denaturation of $94^{\circ} \mathrm{C}$ for $40 \mathrm{sec}$, annealing with $58^{\circ} \mathrm{C}$ for $40 \mathrm{sec}$, extension with $72^{\circ} \mathrm{C}$ for $40 \mathrm{sec}$ and a final extension at $72^{\circ} \mathrm{C}$ for $5 \mathrm{~min}$.) PCR products were visualized on $1.2 \%$ agarose gel along with $1 \mathrm{~kb}$ DNA ladder. The sequencing was done for two virulent isolates. The obtained sequences were then compared with sequences available in Gen Bank by using BLAST server from the NCBI website (http://www.ncbi.nlm.nih.govt/BLAST).

\section{Growth rate of Fusarium $s p$ isolates on different media}

For two virulent isolates, differences in colony growth were observed by growing it in six different media. The different media includes Potato Dextrose Agar media (PDA) (extract of $250 \mathrm{~g}$ of potato, dextrose: $20 \mathrm{~g}$, agar: $20 \mathrm{~g}, 1000 \mathrm{ml}$ of distilled water), Potato Sucrose Agar media (PSA) (extract of $250 \mathrm{~g}$ of potato, sucrose: $20 \mathrm{~g}$, agar: $20 \mathrm{~g}, 1000 \mathrm{ml}$ of distilled water), Oat meal agar media (OMA) (rolled oats: $30 \mathrm{~g}$, agar: $15 \mathrm{~g}, 1000 \mathrm{ml}$ of distilled water), Czapek Dox agar media (CDA) (Sucrose: 30g, $\mathrm{NaNo}_{3}: 2 \mathrm{~g}, \mathrm{~K}_{2} \mathrm{HPo} 4$ : 1g, MgSo $:$ : $1 \mathrm{~g}, \mathrm{KCl}: 0.5 \mathrm{~g}$, FeSo4: 0.01g, Agar: $20 \mathrm{~g}, 1000 \mathrm{ml}$ of distilled water), Malt Extract Agar media (MEA) (malt extract: 25g, agar: $20 \mathrm{~g}, 1000 \mathrm{ml}$ of distilled water) and V8 juice agar media. The colony diameter $(\mathrm{cm})$ was measured at 48, 72, 96, 144 and 168 hours after incubation. The experiment was laid out in completely randomized design with three replications. The colony diameter was measured from all the four sides.

\section{Results and Discussion}

\section{Survey and isolation of Fusarium spp.}

Fusarium wilt of bitter gourd was observed in all the ten locations surveyed and the disease incidence ranged from 11.22 to $52.63 \%$ in all the crop stages (Table.1). The maximum wilt incidence was recorded in Naatukalpalayam field 1 (52.63\%). This was followed by Poosaripalayam, Naatukalpalayam field 2 and Mathampatti. These locations are recorded with wilt incidence of $48.41,45.6,38.2 \%$, respectively. The minimum wilt incidence was recorded in TNAU orchard (11.22\%). The maximum incidence of Fusarium wilt with $52.63 \%$ in Natukalpalayam may be due to presence of higher amount of inoculum in soil or monocropping of bitter gourd repeatedly in same location. A positive 
correlation of inoculum density and disease incidence have been applied to several soil borne diseases caused by Fusarium spp. Marios and Mitchell (1980) has shown that the higher inoculum density of 50,000 chlamydospores per pot resulted in $44 \%$ of crown rot incidence in tomato under glass house condition, whereas pot with minimum inoculum of 500 chlamydospores per pot resulted in $33 \%$ disease incidence only. Ebbels (1975) reported that the cotton wilt caused by $F$. oxysporum $\mathrm{f}$ sp. vasinfectum builds up steadily due to monocropping in Tanzania.

\section{Pathogenicity test}

The plants inoculated with pathogen have shown typical symptoms of yellowing of leaves, drooping and wilting (Plate 2) and the characteristic symptom of vascular discoloration was also observed. Re-isolated the pathogen, which was similar to that of the original culture and thus Koch's postulates were proved. In our study, initial symptom of yellowing was observed as early as 5 DAI in twenty-five days old seedling. This may be due to aggressiveness of pathogen or susceptibility stage of the plant. Cumagun et al. (2010) proposed that the stage or age of the plant may affect the pathogenicity of $F$. oxysporum. In their experiment, symptom was observed on 7 DAI in both 7 days and 30 days old bottle gourd seedlings. However, there is a significant difference in disease severity caused by $F$. oxysporum among 7 days and 30 days old bottle gourd seedlings.

\section{Cultural and morphological characterization}

The results given in Table 2 showed that there is a significant difference among ten isolates with respect to colony characters, pigmentation and colony margin. A white flattened mycelium and reddish pigmentation with smooth margin was observed in BF1. White fluffy mycelial growth with yellowish pigmentation was observed in BF2. BF3 isolate have shown white raised outer growth with pale yellowish pigmentation and smooth margin. Pale yellowish cottony growth and yellowish pigmentation with smooth margin was observed in BF4 isolate. BF5 have shown white raised fluffy mycelial growth with smooth margin. BF6 isolate have shown white sparse mycelial growth with smooth margin and without any pigmentation. Pinkish mycelial growth and pinkish pigmentation was observed in BF8 isolate. The isolate BF9 have shown yellowish fluffy growth without any pigmentation. Whitish mycelial growth without any pigmentation and with smooth margin was observed in BF10 isolate. The front and back view of ten Fusarium isolates plates have shown in the Plate 3. Chandran and Kumar (2012) while studying variability of $F$. solani, they found three isolates as white cottony growth, six as white sparse growth, two as white fluffy and three as dense growth. Similar type of results was also observed by Gogoiet al., (2017).

The colony diameter $(\mathrm{cm})$ of ten Fusarium sp isolates was observed up to seven days (Table 3). On Potato Dextrose Agar medium, the colony diameter ranged from 6.7 to $9.0 \mathrm{~cm}$. The highest colony diameter of $9.0 \mathrm{~cm}$ was observed in BF 2 and BF 7 followed by BF5 $(8.2 \mathrm{~cm})$, BF8 $(7.7 \mathrm{~cm})$, BF9 $(7.4 \mathrm{~cm})$. The minimum colony diameter was observed in BF1 with $6.1 \mathrm{~cm}$. This fast colony growth of the isolates BF2 and BF7 might be due to their virulent nature. Chavanet al.,(2011) recorded cultural and morphological variability of eight different isolates of F.solani causing wilt in Patchouli. They found that the Fusarium isolates with good mycelial growth of $90.00 \mathrm{~mm}$ showed abundant sporulation whereas isolate with mycelial growth of $84.00 \mathrm{~mm}$ showed moderate sporulation. 
Table.1 Survey at different regions of Coimbatore district

\begin{tabular}{|c|c|c|c|c|c|c|}
\hline S.No & Isolate & Location & Latitude $\left({ }^{\circ} \mathbf{N}\right)$ & Longitude $\left({ }^{\circ} \mathbf{E}\right)$ & Crop stage & *PDI (\%) \\
\hline 1. & BF1 & TNAU Orchard & 11.008948 & 76.934065 & Flowering stage & $\begin{array}{c}11.2^{\mathrm{i}} \\
(19.55)\end{array}$ \\
\hline 2. & $\mathrm{BF} 2$ & Naatukalpalayam 1 & 10.6220 & 77.0670 & Harvesting stage & $\begin{array}{c}52.63^{\mathrm{a}} \\
(46.51)\end{array}$ \\
\hline 3. & $\mathrm{BF} 3$ & Naatukalpalayam 2 & 10.6619 & 77.0081 & Harvesting stage & $\begin{array}{c}45.6^{\mathrm{c}} \\
(42.48)\end{array}$ \\
\hline 4. & BF4 & Vadakipalayam & 10.7360 & 76.9641 & Harvesting stage & $\begin{array}{c}33.2^{\mathrm{e}} \\
(35.18)\end{array}$ \\
\hline 5. & BF5 & Vettaikaranputhur & 10.5620 & 76.9211 & Flowering stage & $\begin{array}{c}19.62^{\mathrm{h}} \\
(26.29)\end{array}$ \\
\hline 6. & BF6 & Anaimalai & 10.5821 & 76.9343 & Vegetative stage & $\begin{array}{c}28.3^{\mathrm{f}} \\
(32.14)\end{array}$ \\
\hline 7. & BF7 & Mathampatti & 10.9698 & 76.8598 & Harvesting stage & $\begin{array}{c}38.2^{\mathrm{d}} \\
(38.17)\end{array}$ \\
\hline 8. & BF8 & Annur & 11.2321 & 77.1067 & Harvesting stage & $\begin{array}{c}27.2^{\mathrm{f}} \\
(31.44)\end{array}$ \\
\hline 9. & BF9 & Poosaripalayam & 11.0062 & 76.9333 & Vegetative stage & $\begin{array}{l}48.41^{\mathrm{b}} \\
(44.09)\end{array}$ \\
\hline 10. & BF10 & Pappampatti & 10.9581 & 77.1019 & Flowering stage & $\begin{array}{c}21.3^{\mathrm{g}} \\
(27.49)\end{array}$ \\
\hline \multicolumn{6}{|r|}{ SED } & 0.22 \\
\hline \multicolumn{6}{|r|}{ CD (0.05) } & 0.793 \\
\hline
\end{tabular}

*Mean of three replications; Values in parenthesis are arcsine transformed

In a column, means followed by a common letter are not significantly different at the 5\% level by DMRT

Table.2 Colony characteristics of Fusarium isolates on PDA

\begin{tabular}{|l|l|l|l|l|}
\hline S.No & Isolate & Colony characters $^{\text {a }}$ & Pigmentation $^{\mathbf{b}}$ & Colony margin \\
\hline $\mathbf{1 .}$ & BF1 & White flatten mycelial growth & Whitish & Smooth margin \\
\hline $\mathbf{2 .}$ & BF2 & White fluffy mycelium & Whitish & Irregular margin \\
\hline $\mathbf{3 .}$ & BF3 & $\begin{array}{l}\text { White raised mycelial growth on the outer } \\
\text { border of colony }\end{array}$ & Whitish & Smooth margin \\
\hline $\mathbf{4 .}$ & BF4 & Pale yellowish cottony mycelial growth & Yellowish & Smooth margin \\
\hline $\mathbf{5 .}$ & BF5 & White raised fluffy growth & Light yellow & Smooth margin \\
\hline $\mathbf{6 .}$ & BF6 & White sparse mycelial growth & Whitish & Smooth margin \\
\hline $\mathbf{7 .}$ & BF7 & White cottony growth & Whitish & Smooth margin \\
\hline $\mathbf{8 .}$ & BF8 & Pinkish mycelial growth & Pinkish & Irregular margin \\
\hline $\mathbf{9 .}$ & BF9 & Yellowish fluffy mycelium & Whitish & Irregular margin \\
\hline $\mathbf{1 0 .}$ & BF10 & Whitish mycelium with raised border & Whitish & Smooth margin \\
\hline \multicolumn{2}{r}{${ }^{\text {a } C o l o n y ~ c h a r a c t e r s ~ w e r e ~ d e t e r m i n e d ~ b y ~ o b s e r v i n g ~ t h e ~ u p p e r ~ s u r f a c e ~ o f ~ t h e ~ c o l o n y ~}$} & \\
\hline
\end{tabular}


Table.3 Colony diameter (cm) of Fusarium isolates on PDA for 7 days of incubation

\begin{tabular}{|c|c|c|c|c|c|c|c|}
\hline \multirow[t]{2}{*}{ S.No } & \multirow[t]{2}{*}{ Isolates } & \multicolumn{6}{|c|}{ *Colony diameter $(\mathrm{cm})$} \\
\hline & & $48 \mathrm{hrs}$ & $72 \mathrm{hrs}$ & $96 \mathrm{hrs}$ & $120 \mathrm{hrs}$ & $144 \mathrm{hrs}$ & $168 \mathrm{hrs}$ \\
\hline 1. & BF1 & $2.6^{\mathrm{e}}$ & $3.1^{\mathrm{f}}$ & $4.1^{\mathrm{i}}$ & $5.2^{\mathrm{g}}$ & $5.6^{\mathrm{f}}$ & $6.1^{\mathrm{h}}$ \\
\hline 2. & $\mathrm{BF} 2$ & $2.7^{\mathrm{d}}$ & $4.2^{\mathrm{c}}$ & $6.2^{\mathrm{b}}$ & $8.1^{\mathrm{a}}$ & $8.6^{\mathrm{a}}$ & $9.0^{\mathrm{a}}$ \\
\hline 3. & BF3 & $2.6^{\mathrm{e}}$ & $3.4^{\mathrm{e}}$ & $4.3^{\mathrm{h}}$ & $5.6^{\mathrm{f}}$ & $6.1^{\mathrm{e}}$ & $6.7^{\mathrm{g}}$ \\
\hline 4. & $\mathrm{BF} 4$ & $2.1^{\mathrm{f}}$ & $3.2^{\mathrm{f}}$ & $4.6^{\mathrm{g}}$ & $5.6^{\mathrm{t}}$ & $6.6^{\mathrm{d}}$ & $7.1^{\mathrm{ef}}$ \\
\hline 5. & BF5 & $3.5^{\mathrm{b}}$ & $4.6^{\mathrm{b}}$ & $5.6^{\mathrm{d}}$ & $6.7^{\mathrm{d}}$ & $7.7^{\mathrm{b}}$ & $8.2^{\mathrm{b}}$ \\
\hline 6. & BF6 & $2.7^{\mathrm{de}}$ & $3.6^{\mathrm{d}}$ & $5.1^{\mathrm{e}}$ & $6.1^{\mathrm{e}}$ & $6.6^{\mathrm{d}}$ & $7.2 \mathrm{~d}^{\mathrm{e}}$ \\
\hline 7. & BF7 & $3.1^{\mathrm{c}}$ & $4.1^{\mathrm{c}}$ & $6.3^{\mathrm{b}}$ & $7.6^{\mathrm{b}}$ & $8.4^{\mathrm{a}}$ & $9.0^{\mathrm{a}}$ \\
\hline 8. & BF8 & $4.1^{\mathrm{a}}$ & $5.6^{\mathrm{a}}$ & $6.6^{\mathrm{a}}$ & $7.1^{\mathrm{c}}$ & $7.5^{\mathrm{b}}$ & $7.7^{\mathrm{c}}$ \\
\hline 9. & BF9 & $3.2^{\mathrm{c}}$ & $4.6^{\mathrm{b}}$ & $5.8^{\mathrm{c}}$ & $6.6^{\mathrm{d}}$ & $7.1^{\mathrm{c}}$ & $7.4^{\mathrm{d}}$ \\
\hline 10. & BF10 & $2.6^{\mathrm{e}}$ & $3.6^{\mathrm{d}}$ & $4.9^{f}$ & $6.1^{\mathrm{e}}$ & $6.6^{\mathrm{d}}$ & $6.9^{\mathrm{fg}}$ \\
\hline \multicolumn{2}{|c|}{$\operatorname{SEm}( \pm)$} & 0.03 & 0.04 & 0.21 & 0.23 & 0.10 & 0.76 \\
\hline \multicolumn{2}{|c|}{$\mathrm{CD}(0.05)$} & 0.10 & 0.17 & 0.20 & 0.23 & 0.36 & 0.56 \\
\hline
\end{tabular}

*values are mean of three replications

In column, means followed by a common letter are not significantly different at the $5 \%$ level by DMRT

Table.4 Morphological characteristics of Fusarium isolates on PDA

\begin{tabular}{|c|c|c|c|c|c|c|c|c|}
\hline \multirow[t]{2}{*}{ S.No } & \multirow[t]{2}{*}{ Isolate } & \multicolumn{2}{|c|}{ Spore size $(\mu \mathrm{m})$} & \multicolumn{2}{|c|}{ Septations } & \multicolumn{2}{|c|}{ Shape } & \multirow[t]{2}{*}{ Colour } \\
\hline & & $\begin{array}{l}\text { Micro } \\
\text { conidia } \\
\text { (L X B) }\end{array}$ & $\begin{array}{l}\text { Macro } \\
\text { conidia } \\
\text { (L X B) }\end{array}$ & $\begin{array}{l}\text { Micro } \\
\text { conidia }\end{array}$ & $\begin{array}{l}\text { Macro } \\
\text { conidia }\end{array}$ & $\begin{array}{l}\text { Micro } \\
\text { conidia }\end{array}$ & $\begin{array}{l}\text { Macro } \\
\text { conidia }\end{array}$ & \\
\hline 1. & BF1 & $\begin{array}{l}(8.158- \\
11.109) \\
\quad X \\
(3.453- \\
4.405)\end{array}$ & $\begin{array}{l}(13.471- \\
21.440) \\
X \\
(4.604- \\
5.122)\end{array}$ & 0 & $1-2$ & $\begin{array}{l}\text { Elongated } \\
\text { oval }\end{array}$ & $\begin{array}{l}\text { Elongated, } \\
\text { curved } \\
\text { with blunt } \\
\text { ends }\end{array}$ & Hyaline \\
\hline 2. & BF2 & $\begin{array}{l}(8.984- \\
10.127) \\
\quad X \\
(2.807- \\
4.005)\end{array}$ & $\begin{array}{l}(13.927- \\
22.768) \\
X \\
(4.604- \\
4.883)\end{array}$ & 0 & $3-4$ & $\begin{array}{l}\text { Elongated } \\
\text { oval }\end{array}$ & $\begin{array}{l}\text { Sickle } \\
\text { shaped }\end{array}$ & Hyaline \\
\hline 3. & BF3 & $\begin{array}{l}(8.718- \\
11.449) \\
X \\
(3.311- \\
4.441)\end{array}$ & $\begin{array}{l}(14.368- \\
19.2) \\
\quad X \\
(3.883- \\
6.45)\end{array}$ & 0 & $3-4$ & $\begin{array}{l}\text { Elongated } \\
\text { oval }\end{array}$ & $\begin{array}{l}\text { Sickle } \\
\text { shaped }\end{array}$ & Hyaline \\
\hline
\end{tabular}




\begin{tabular}{|c|c|c|c|c|c|c|c|c|}
\hline 4. & BF4 & $\begin{array}{l}(8.857- \\
11.955) \\
\quad X \\
(2.2884- \\
4.205)\end{array}$ & $\begin{array}{l}(13.483- \\
16.00) \\
X \\
(4.000- \\
5.215)\end{array}$ & 0 & $1-2$ & $\begin{array}{l}\text { Elongated } \\
\text { oval }\end{array}$ & $\begin{array}{l}\text { Elongated, } \\
\text { curved } \\
\text { with blunt } \\
\text { ends }\end{array}$ & Hyaline \\
\hline 5. & BF5 & $\begin{array}{l}(10.511- \\
14.843) \\
X \\
(3.124- \\
5.200)\end{array}$ & $\begin{array}{l}(19.349- \\
33.970) \\
\quad X \\
(3.256- \\
4.94)\end{array}$ & 0 & $3-4$ & $\begin{array}{l}\text { Elongated } \\
\text { oval }\end{array}$ & $\begin{array}{l}\text { Sickle } \\
\text { shaped } \\
\text { with } \\
\text { narrow tip }\end{array}$ & Hyaline \\
\hline 6. & BF6 & $\begin{array}{l}(8.718- \\
10.332) \\
X \\
(3.162- \\
4.561)\end{array}$ & $\begin{array}{l}(13.220- \\
22.768) \\
X \\
(3.720- \\
4.205)\end{array}$ & 0 & $1-3$ & $\begin{array}{l}\text { Round to } \\
\text { oval }\end{array}$ & $\begin{array}{l}\text { Sickle } \\
\text { shaped } \\
\text { with blunt } \\
\text { ends }\end{array}$ & Hyaline \\
\hline 7. & BF7 & $\begin{array}{l}(10.560- \\
12.265) \\
\quad X \\
(3.538- \\
3.842)\end{array}$ & $\begin{array}{l}(17.839- \\
23.923) \\
X \\
(4.870- \\
5.385)\end{array}$ & 0 & $2-3$ & $\begin{array}{l}\text { Elongated } \\
\text { oval }\end{array}$ & $\begin{array}{l}\text { Sickle } \\
\text { shaped }\end{array}$ & Hyaline \\
\hline 8. & BF8 & $\begin{array}{l}(8.352- \\
11.207) \\
X \\
(3.418- \\
6.512)\end{array}$ & $\begin{array}{l}(14.403- \\
19.251) \\
X \\
(4.079- \\
5.492)\end{array}$ & 0 & $1-2$ & $\begin{array}{l}\text { Elongated } \\
\text { oval }\end{array}$ & $\begin{array}{l}\text { Elongated, } \\
\text { curved } \\
\text { with blunt } \\
\text { ends }\end{array}$ & Hyaline \\
\hline 9. & BF9 & $\begin{array}{l}(9.159- \\
11.705) \\
X \\
(4.317- \\
5.492)\end{array}$ & $\begin{array}{l}(19.042- \\
31.902) \\
\quad X \\
(2.332- \\
4.079)\end{array}$ & 0 & $3-4$ & $\begin{array}{l}\text { Elongated } \\
\text { oval }\end{array}$ & $\begin{array}{l}\text { Sickle } \\
\text { shaped }\end{array}$ & Hyaline \\
\hline 10. & BF10 & $\begin{array}{l}(10.024- \\
12.758) \\
\quad X \\
(2.332- \\
3.795)\end{array}$ & $\begin{array}{l}(17.533- \\
32.423) \\
\quad X \\
(2.778- \\
4.299)\end{array}$ & 0 & 3 & Oval & $\begin{array}{l}\text { Sickle } \\
\text { shaped } \\
\text { with } \\
\text { pointed tip }\end{array}$ & Hyaline \\
\hline
\end{tabular}


Table.5a Colony diameter of Fusarium isolate BF2 for 7 days of incubation on different media

\begin{tabular}{|l|r|r|r|r|r|r|}
\hline \multirow{2}{*}{ Media } & \multicolumn{6}{|c|}{$*$ Colony diameter (cm) } \\
\cline { 2 - 8 } & $\mathbf{4 8} \mathbf{~ h r s}$ & $\mathbf{7 2} \mathbf{~ h r s}$ & $\mathbf{9 6} \mathbf{~ h r s}$ & $\mathbf{1 2 0} \mathbf{~ h r s}$ & $\mathbf{1 4 4} \mathbf{~ h r s}$ & $\mathbf{1 6 8} \mathbf{~ h r s}$ \\
\hline PDA & $2.7^{\mathrm{c}}$ & $4.2^{\mathrm{a}}$ & $6.2^{\mathrm{a}}$ & $8.1^{\mathrm{a}}$ & $8.6^{\mathrm{a}}$ & $9.0^{\mathrm{a}}$ \\
\hline PSA & $2.6^{\mathrm{c}}$ & $4.3^{\mathrm{a}}$ & $6.1^{\mathrm{a}}$ & $7.6^{\mathrm{b}}$ & $8.6^{\mathrm{a}}$ & $9.0^{\mathrm{a}}$ \\
\hline CPA & $3.4^{\mathrm{a}}$ & $3.9^{\mathrm{b}}$ & $4.6^{\mathrm{b}}$ & $5.1^{\mathrm{d}}$ & $5.9^{\mathrm{c}}$ & $6.4^{\mathrm{cd}}$ \\
\hline OMA & $3.0^{\mathrm{b}}$ & $3.7^{\mathrm{c}}$ & $4.4^{\mathrm{c}}$ & $5.2^{\mathrm{d}}$ & $5.8^{\mathrm{c}}$ & $6.6^{\mathrm{c}}$ \\
\hline V8 & $2.7^{\mathrm{c}}$ & $4.0^{\mathrm{b}}$ & $4.6^{\mathrm{b}}$ & $5.8^{\mathrm{c}}$ & $6.3^{\mathrm{b}}$ & $7.2^{\mathrm{b}}$ \\
\hline MEA & $1.8^{\mathrm{d}}$ & $2.7^{\mathrm{d}}$ & $3.6^{\mathrm{d}}$ & $4.4^{\mathrm{e}}$ & $5.1^{\mathrm{d}}$ & $6.2^{\mathrm{d}}$ \\
\hline SEm( $\mathbf{( \pm}$ & 0.04 & 0.04 & 0.05 & 0.09 & 0.06 & 0.10 \\
\hline CD $(\mathbf{0 . 0 5})$ & 0.132 & 0.136 & 0.194 & 0.322 & 0.229 & 0.369 \\
\hline
\end{tabular}

*values are mean of three replications

In column, means followed by a common letter are not significantly different at the $5 \%$ level by DMRT

Table.5b Colony diameter of Fusarium isolate BF7 for 7 days of incubation on different

\begin{tabular}{|c|c|c|c|c|c|c|}
\hline \multirow[t]{2}{*}{ Media } & \multicolumn{6}{|c|}{ *Colony diameter (cm) } \\
\hline & $48 \mathrm{hrs}$ & $72 \mathrm{hrs}$ & $96 \mathrm{hrs}$ & $120 \mathrm{hrs}$ & 144 hrs & $168 \mathrm{hrs}$ \\
\hline PDA & $3.1^{\mathrm{a}}$ & $4.1^{\mathrm{b}}$ & $6.3^{\mathrm{a}}$ & $7.6^{\mathrm{b}}$ & $8.4^{\mathrm{a}}$ & $9.0^{\mathrm{a}}$ \\
\hline PSA & $3.2^{\mathrm{a}}$ & $4.6^{\mathrm{a}}$ & $6.2^{\mathrm{a}}$ & $8.2^{\mathrm{a}}$ & $8.7^{\mathrm{a}}$ & $9.0^{\mathrm{a}}$ \\
\hline CPA & $2.4^{\mathrm{c}}$ & $3.6^{\mathrm{c}}$ & $4.7^{\mathrm{b}}$ & $5.9^{\mathrm{c}}$ & $7.5^{\mathrm{b}}$ & $8.8^{\mathrm{a}}$ \\
\hline OMA & $2.6^{\mathrm{b}}$ & $3.7^{\mathrm{c}}$ & $4.6^{\mathrm{b}}$ & $5.7^{\mathrm{c}}$ & $7.7^{\mathrm{b}}$ & $8.0^{\mathrm{b}}$ \\
\hline V8 & $2.1^{\mathrm{d}}$ & $3.1^{\mathrm{d}}$ & $4.4^{\mathrm{c}}$ & $5.7^{\mathrm{c}}$ & $6.2^{\mathrm{c}}$ & $6.5^{\mathrm{d}}$ \\
\hline MEA & $1.4^{\mathrm{e}}$ & $3.2^{\mathrm{d}}$ & $4.2^{\mathrm{d}}$ & $5.3^{\mathrm{d}}$ & $6.5^{\mathrm{c}}$ & $7.6^{\mathrm{c}}$ \\
\hline $\operatorname{SEm}( \pm)$ & 0.03 & 0.05 & 0.03 & 0.06 & 0.10 & 0.11 \\
\hline CD (0.05) & 0.124 & 0.182 & 0.128 & 0.207 & 0.376 & 0.408 \\
\hline
\end{tabular}

*values are mean of three replications

In column, means followed by a common letter are not significantly different at the $5 \%$ level by DMRT 
Plate.1a Overview of Infected field b. Yellowing of leaves c. Vascular discoluration

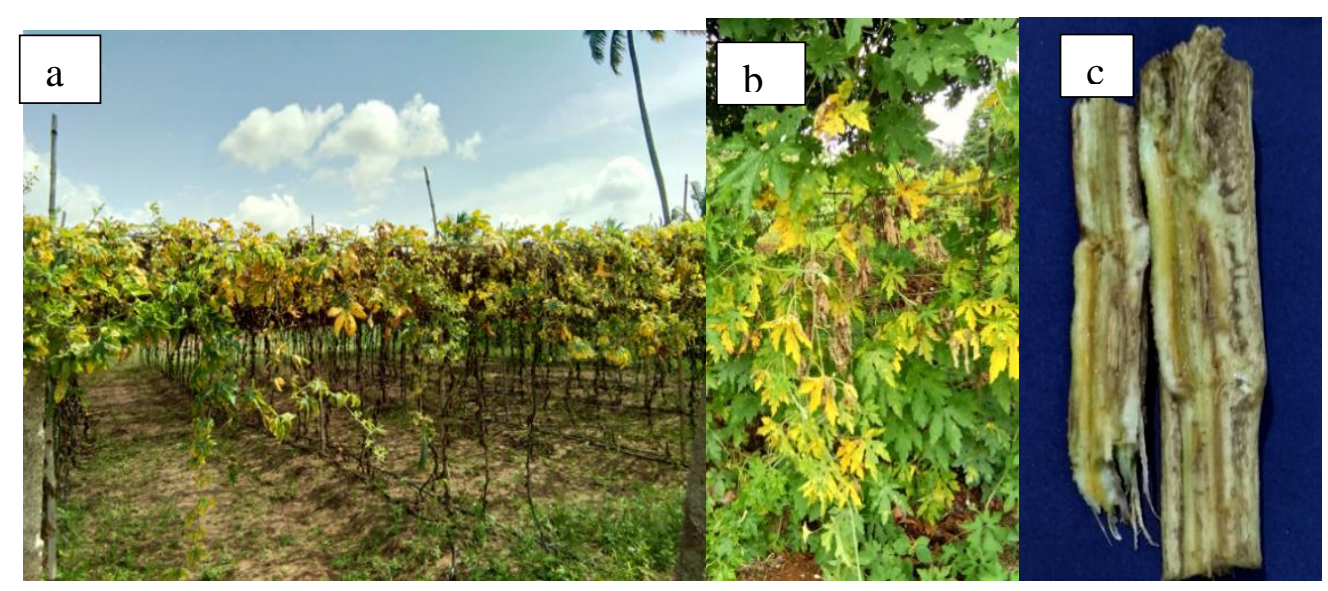

Plate.2 Pathogenicity test (a. Control, b. Inoculated plant shown symptom)
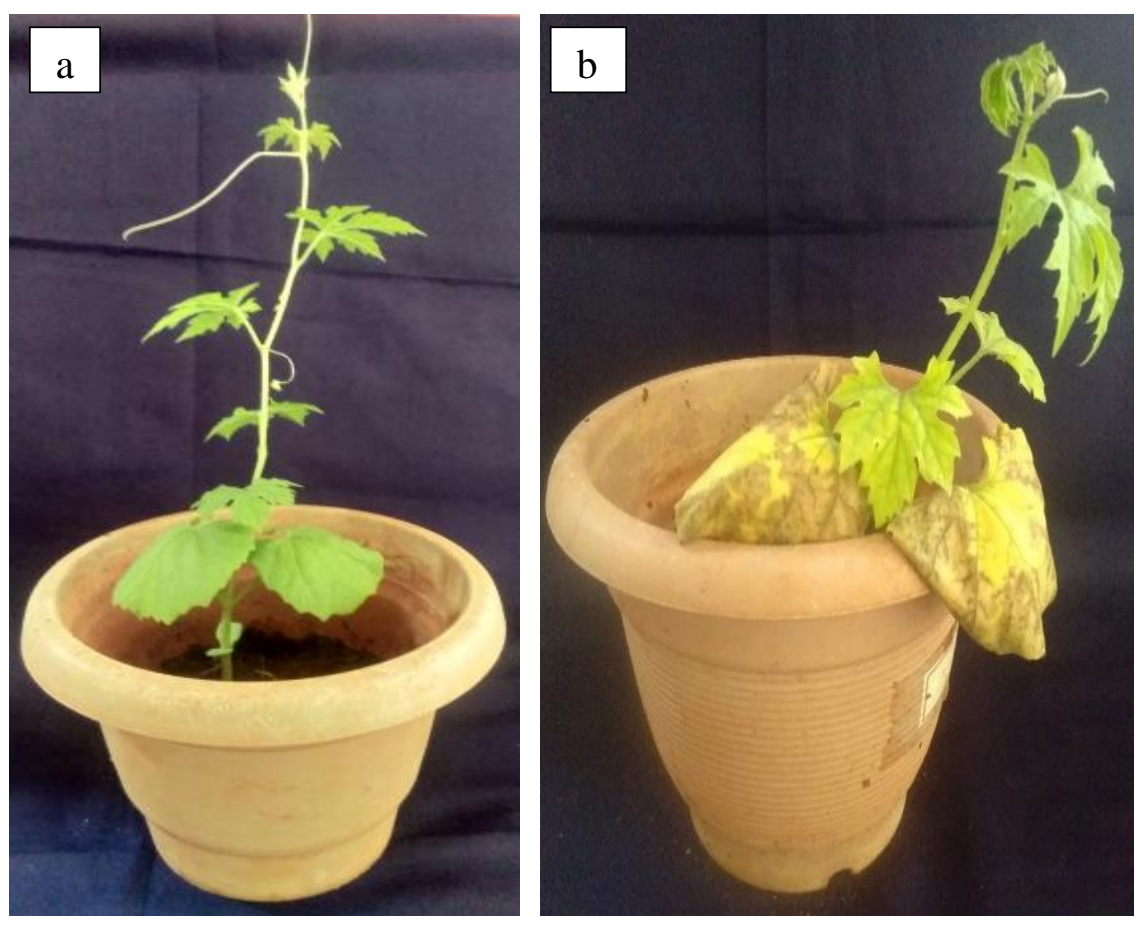
Plate.3 Pure culture of Fusarium isolates on PDA

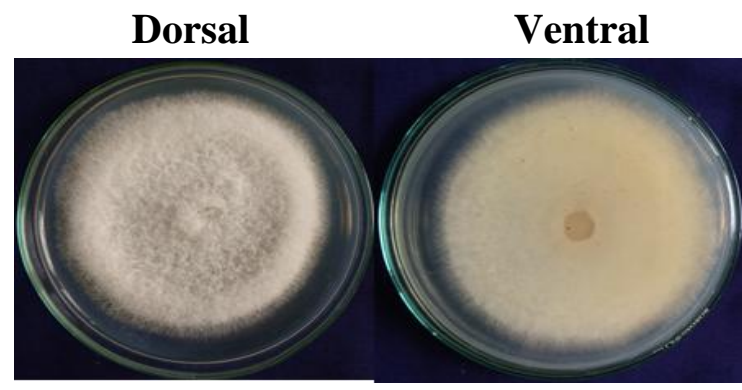

BF 1

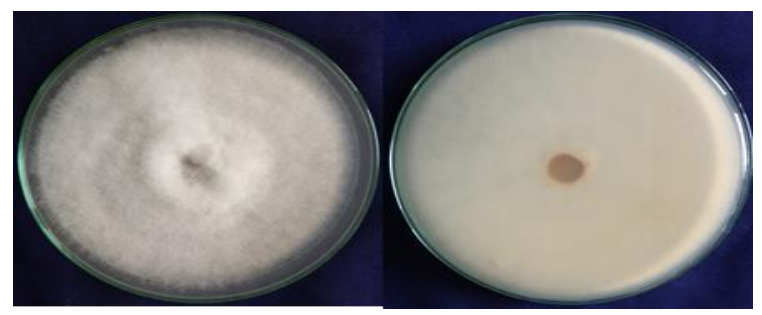

BF 2

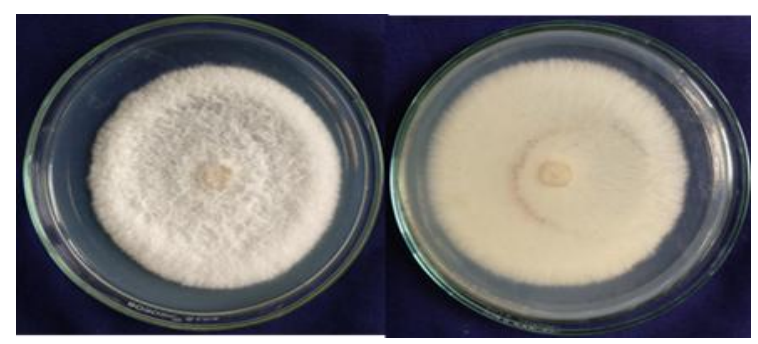

BF 3

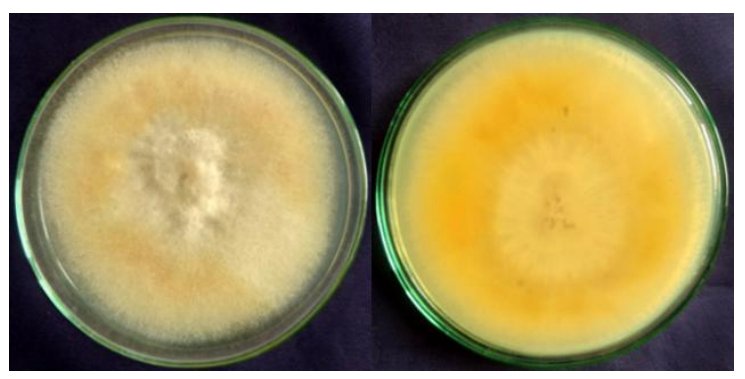

BF 4

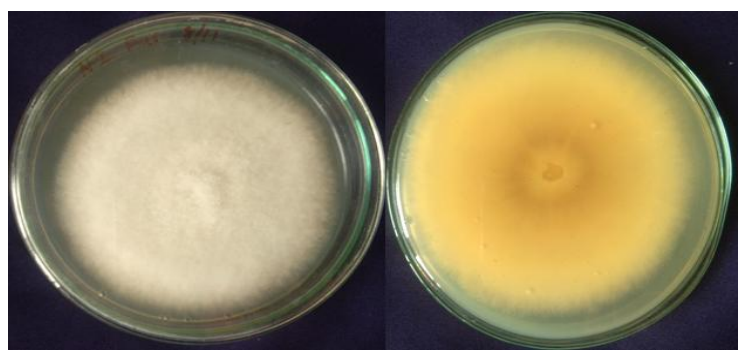

BF 5

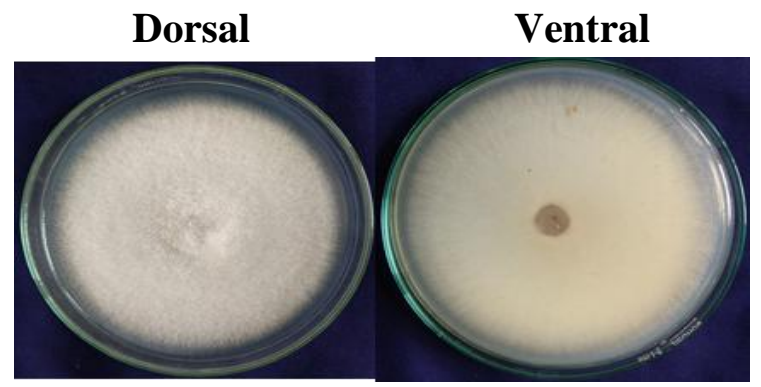

BF 6

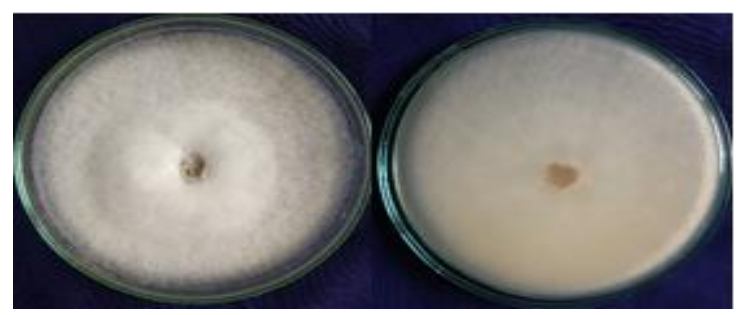

BF7

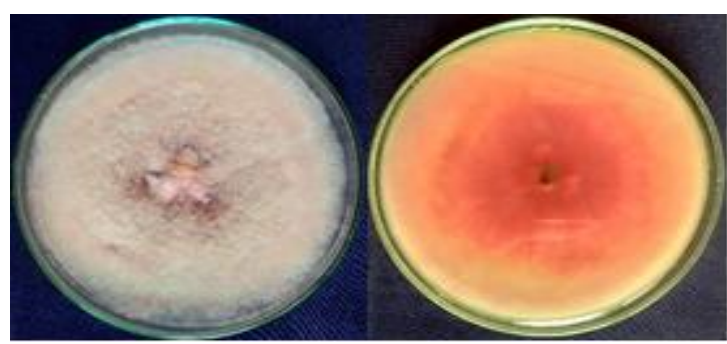

BF 8

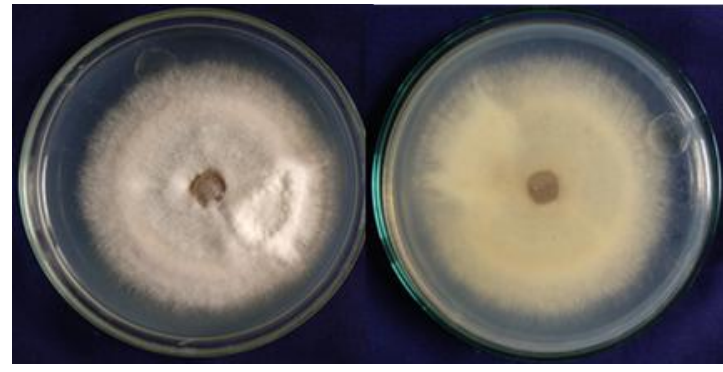

BF 9

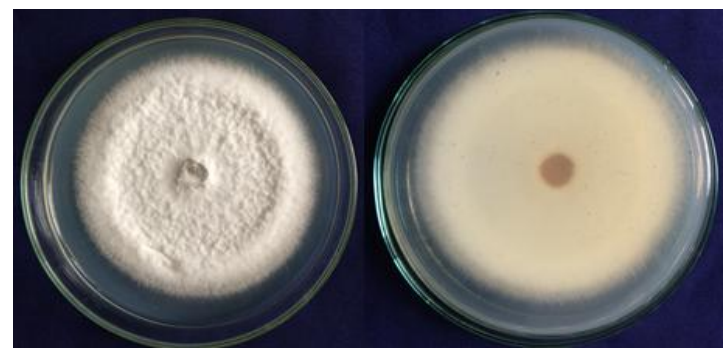

BF 10 
Plate.4 Photomicrograph showing micro and macroconidia of Fusarium isolates (40X)

Microconidia

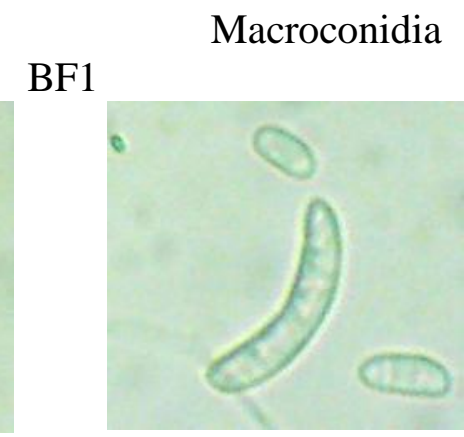

BF2

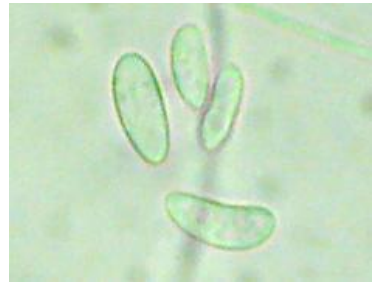

\section{$\mathrm{BF} 3$}

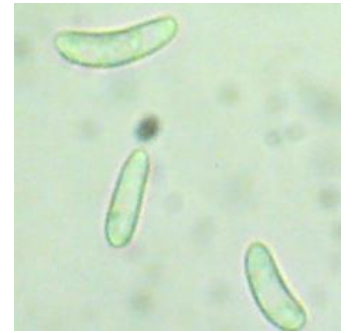

\section{BF4}

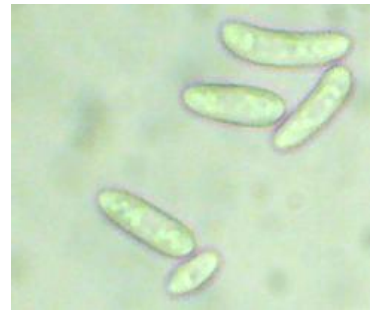

\section{BF5}
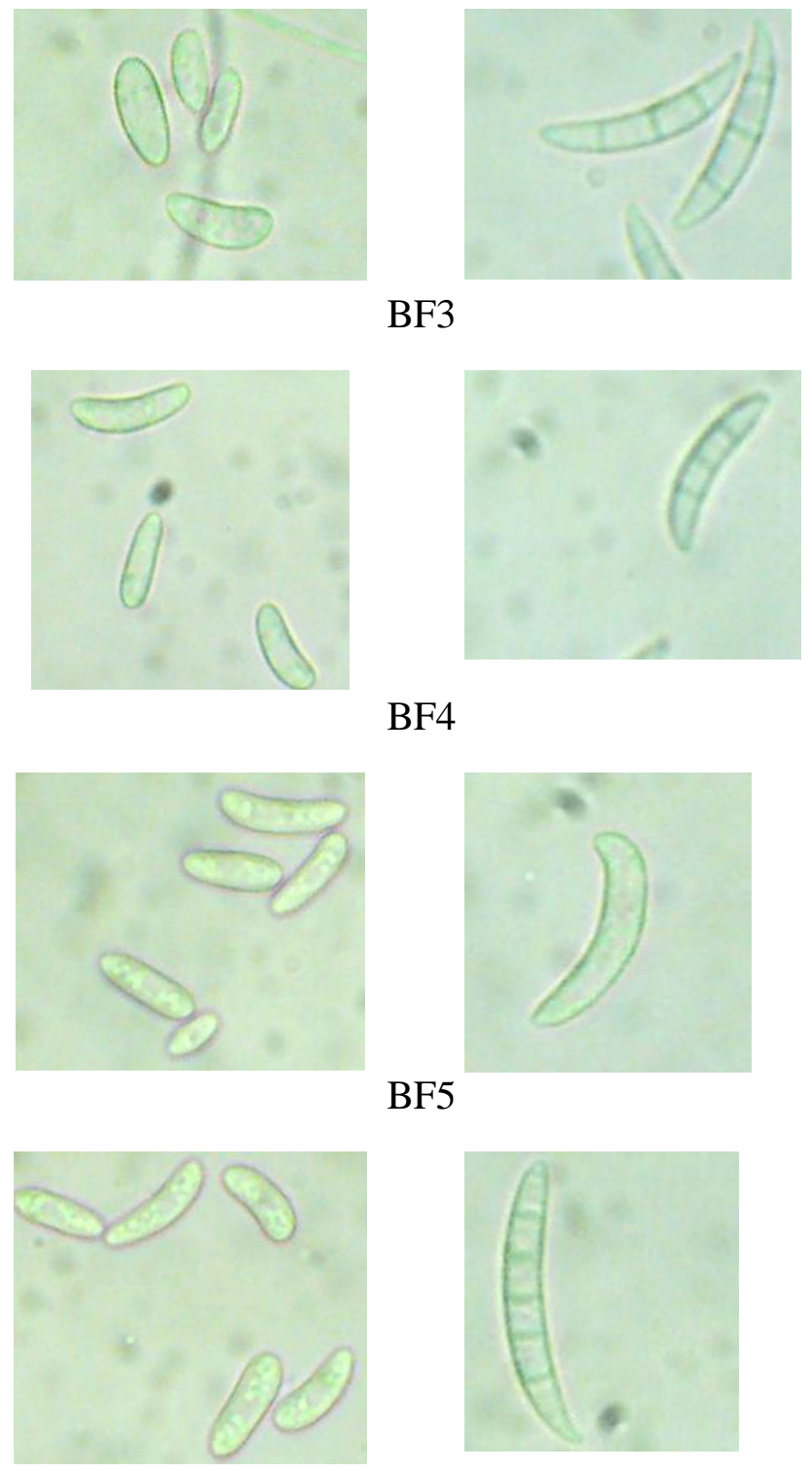

Microconidia

BF6 Macroconidia
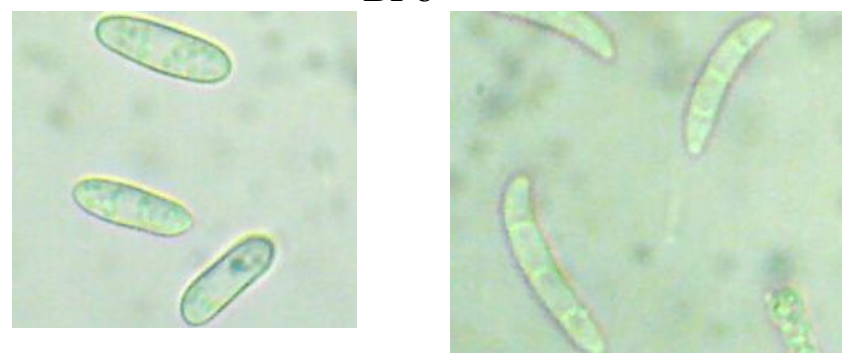

BF7

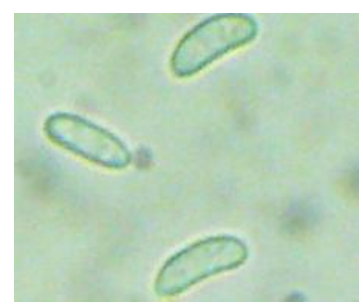

BF8

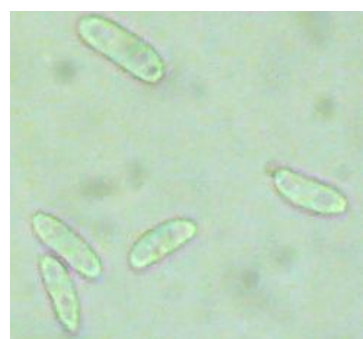

BF9

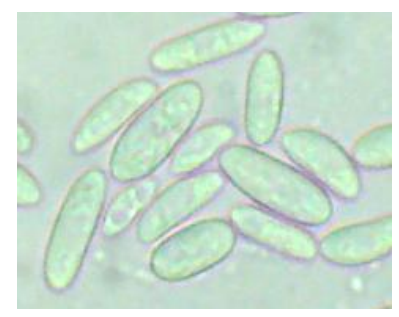

BF10
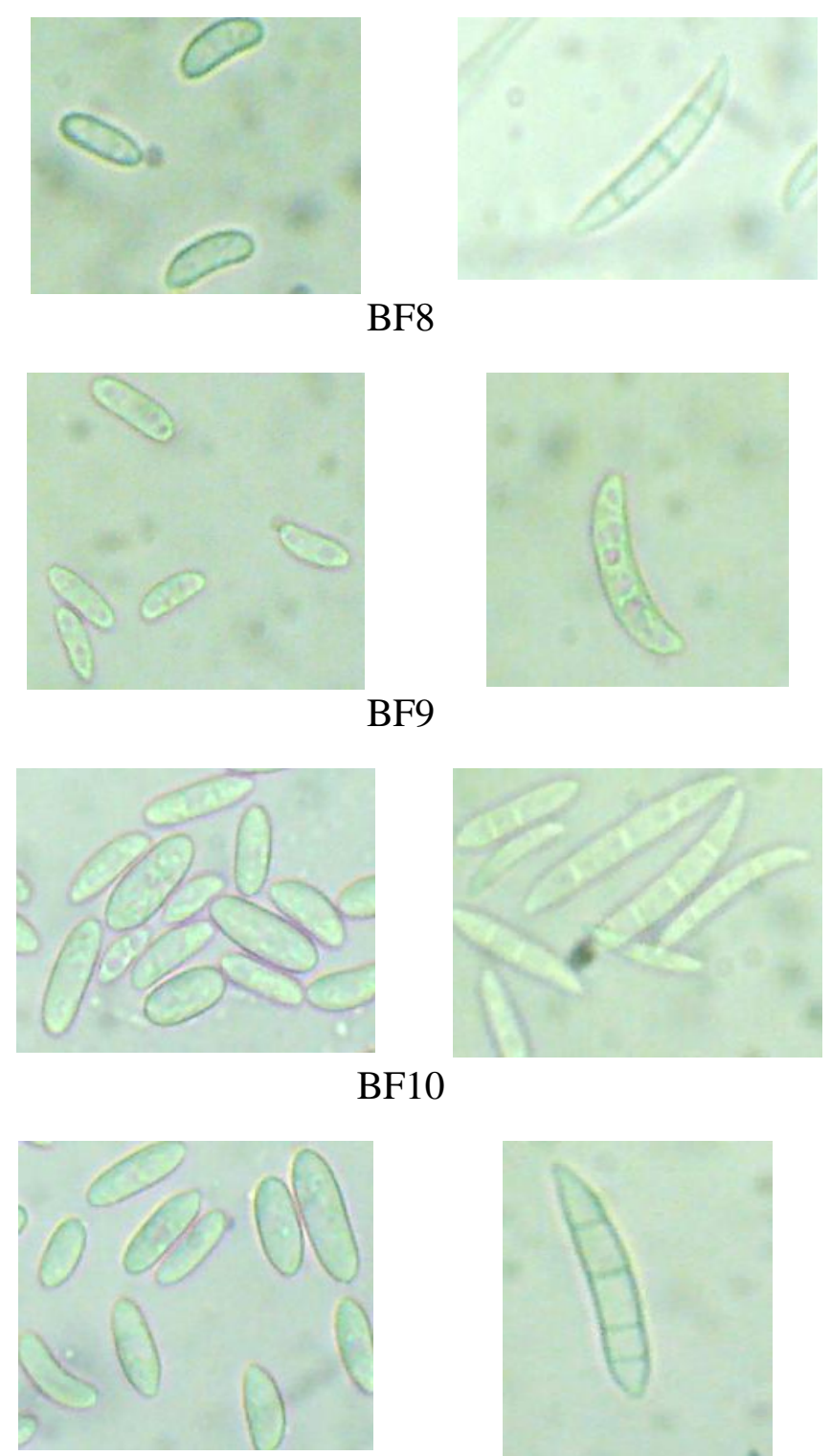

\section{B10}

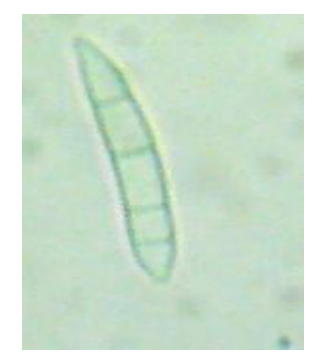


Plate.5 (a and b) Photomicrograph showing terminal and intercalary chlamydospore (40X)

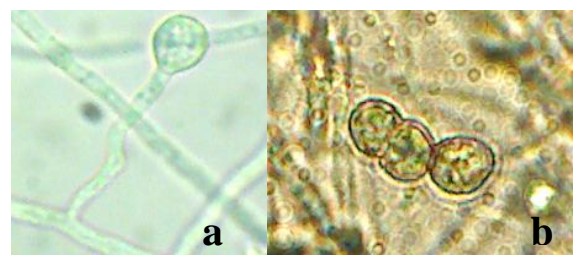

Plate.6 Molecular characterisation of Fusarium isolates

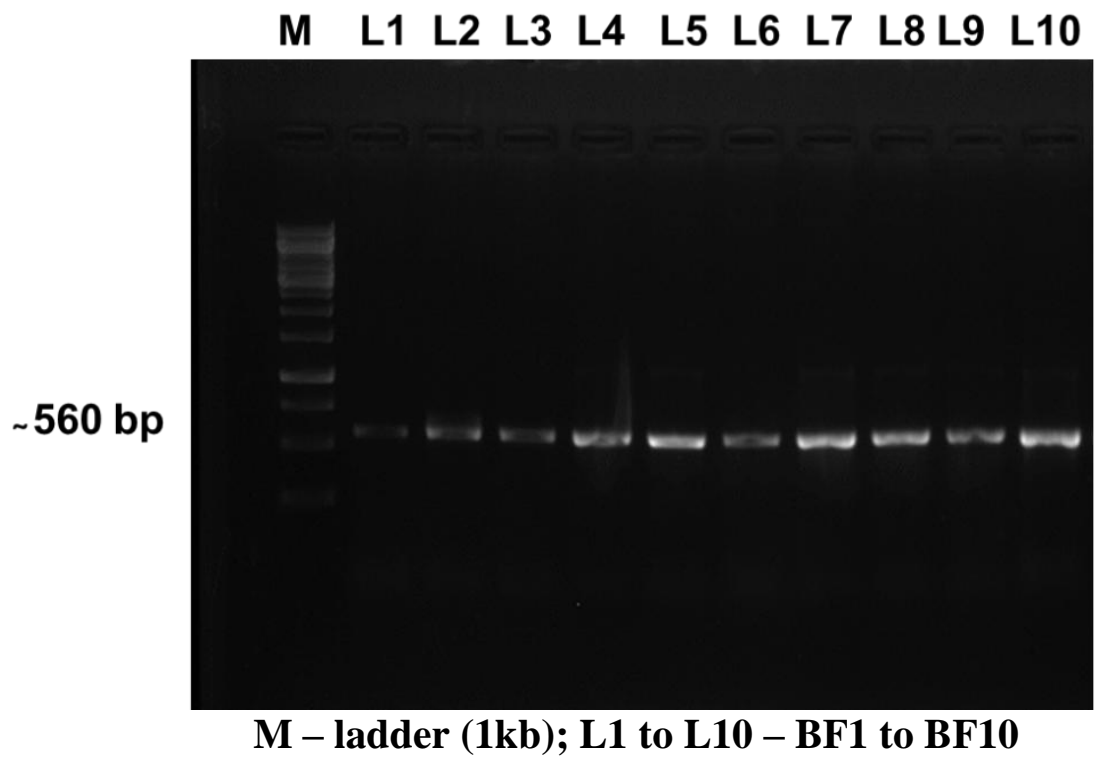

Fusarium spp produced two types of asexual spores viz, microconidia and macroconidia and a resting spore namely chlamydospores in 15-20 days old cultures. The images of micro and macroconidia of all isolates were given in Plate 4. Chlamydospores were observed in end of the mycelium as terminal chlamydospores (Plate 5a) and also in the middle as intercalary chlamydospores (Plate $5 b)$ in chain. The size, shape and number of septation in micro and macroconidia of all the ten isolates were given in Table 4. There is no septation in microconidia and 2-4 septations in macroconidia. The shape of microconidia is elongated oval and hyaline. The shape of macroconidia is varied for different isolates. The isolates BF1, BF4 and BF8 had elongated and curved macroconidia with blunt ends. Whereas, the isolates BF2, BF3, BF7 and BF9 have sickle shaped macroconidia. The other isolates namely BF5 and BF10 have sickle shaped with pointed tips. The size of microconidia ranged from $(9.2-11.8) \times(3.2-$ 4.6) $\mu \mathrm{m}$ and macroconidia is (15.7-24.4) $\mathrm{x}(3.8$ $-5.0) \mu \mathrm{m}$. The maximum size of microconidia was observed in isolates BF5 (10.511 $14.843) \times(3.124-5.200) \mu \mathrm{m}$ which is followed by BF10 $(10.024-12.758) \times(2.332$

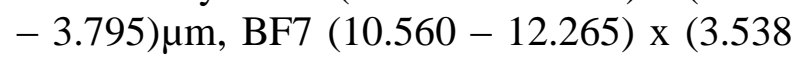

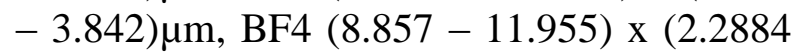
$-4.205) \mu \mathrm{m}$ and minimum size was observed in BF2 (8.984 - 10.127) x (2.807 - 4.005) $\mu \mathrm{m}$. The maximum size of macroconidia was observed in isolate BF5 (19.349 - 33.970) $\mathrm{x}$ $(3.256-4.94) \mu \mathrm{m}$ which is followed by BF10 $(17.533-32.423) \times(2.778-4.299) \mu \mathrm{m}, \mathrm{BF} 9$ $(19.042-31.902) \times(2.332-4.079) \mu \mathrm{m}, \mathrm{BF} 7$ (17.839- 23.923) x $(4.870-5.385) \mu \mathrm{m}$ and minimum size of macroconidia was observed in BF4 (13.483 - 16.00) x (4.000 - 5.215) 
$\mu \mathrm{m}$. Gogoi et al.(2017) observed that the size of micro and macroconidia of Fusarium solani ranges from (3-4 x 1-2) to $(9-10 \times 1-3)$ $\mu \mathrm{m}$ and $(26.42-32.46) \times(3.24-4.74) \mu \mathrm{m}$ to $(17.39-23.17) \times(2.91-4.51) \mu \mathrm{m}$ respectively. Chandran et al. (2012) studied the morphological variability of $F$. solani. They observed 3-5 and 0-1 septations in macro and microconidia respectively, which is similar to our results. They also given that the macroconidia are sickle shaped with blunt ends and microconidia areround to oval shaped. Results obtained in our observation is in agreement with the work mentioned above.

\section{Molecular characterization}

The fungal pathogen associated with bitter gourd wilt was confirmed using universal ITS primers. The PCR amplification of ITS region of Fusarium spp., yielded an amplicon size of approximately 560 base pairs (Plate 6). The sequence analysis of BF2 and BF7 has shown 99\% and $98 \%$ similarity with Fusarium solani, respectively. The amplicon of BF2 and BF7 was sequenced and submitted to Gene bank. These results are in agreement with the findings of Sreegayathri et al.,(2018) who reported that the PCR amplification of ITS region of Fusarium solani yielded approximately 550 to 570 bp amplicon

\section{Growth rate on different media}

The growth rate of two virulent isolates $\mathrm{BF} 2$ and BF7 on different media was studied seven days after incubation (Table $5 \mathrm{a}$ and $5 \mathrm{~b}$ ). The mycelial growth of isolates $\mathrm{BF} 2$ and $\mathrm{BF} 7$ differ statistically in different media with mean value ranged from 6.2 to $9.0 \mathrm{~cm}$ and 6.5 to $9.0 \mathrm{~cm}$ at room temperature, respectively. For the isolate BF2, the maximum growth of $9 \mathrm{~cm}$ was observed in Potato Dextrose Agar media (PDA) which is on par with Potato Sucrose agar (PSA) media $(9.0 \mathrm{~cm})$ and it is followed by V8 $(7.2 \mathrm{~cm})$, OMA $(6.6 \mathrm{~cm})$ and
CPA $(6.4 \mathrm{~cm})$ whereas minimum growth of $6.2 \mathrm{~cm}$ was observed in MEA. For the isolate BF7, maximum growth of $9 \mathrm{~cm}$ was recorded in PSA which is on par with PDA $(9.0 \mathrm{~cm})$ and it is followed by CPA $(8.8 \mathrm{~cm})$, OMA $(8.0 \mathrm{~cm})$ and MEA $(7.6 \mathrm{~cm})$ whereas minimum growth of $6.5 \mathrm{~cm}$ was recorded in V8 juice agar media. Similar type of work was done by (Mezzomo et al., 2018). This maximum rate of mycelial growth in PDA and PSA medium might be due to greater utilization of carbohydrate source by the Fusarium sp. Silva and Teixeira (2012) state that carbohydrate-richmedia such as PDA and PSA can induce the production of conidia of F. solani. Lazarotto et al. (2013) also suggested PDA and PSA medium for the mass production of macroconidia of $F$. chlamydosporum.

The results of the present study concluded that Fusarium solani, a fungus was associated with bitter gourd wilt disease. The identity of the fungus was confirmed through cultural, morphological and molecular characterization through sequence comparison of ITS region using BLAST search. The variations were observed in cultural and morphological characters among F.solani isolates. The culture media such as PDA and PSA supported the growth of fungus.

\section{Acknowledgement}

The authors are thankful to the Director, CPPS, Head of the Department and Professor of Department of Plant Pathology, TNAU, Coimbatore for providing support, guidance and financial assistance. The author would like to acknowledge DST-FIST and UGCSAP-DRSI for providing facilities at Department of Plant Pathology.

\section{References}

Chandran, M Ravi, and M Reddi Kumar. 2012. "Studies on Cultural, 
Morphological Variability in Isolates of Fusarium Solani (Mart.) Sacc., Incitant of Dry Root-Rot of Citrus" 6 (2): 152 62.

Chavan, Sreedevi S, Yashoda R Hegde, and S K Prashanthi. 2011. "Morphological and Molecular Variability in Fusarium Solani Causing Wilt of Patchouli" 64 (3): 25860.

Cumagun, C.J.R., Aguirre, J.A., Relevante, C.A. and Balatero, C.H., 2010. Pathogenicity and aggressiveness of Fusarium oxysporum Schl. in bottle gourd and bitter gourd. Plant Protection Science, 46(2), pp.51-58.

Ebbels, D.L., 1975. Fusarium wilt of cotton: a review, with special reference to Tanzania. Cotton Growing Review, 52(4), pp.295-339.

Gogoi, Monuj, D.K. Sarmah, and S. Ali. 2017. "Cultural and Morphological Variations of Fusarium Solani (Mart.) Sacc. Causing Root Rot of Patchouli in Assam, India." International Journal of Current Microbiology and Applied Sciences 6 (11): 1889 1901.https://doi.org/10.20546/ijcmas.201 7.611.225.

Lazarotto, M.; Mezzomo, R.; Maciel, C. G.; Finger, G.; Muniz, M.F.B. 2014. Mycelia growth and sporulation of Fusarium chlamydosporum species complex under different culture conditions. Revista Ciências. Agrárias, v. 57, n. 1, p. 35-40.

Marois, J. J., \& Mitchell, D. J., 1981. Effects of fumigation and fungal antagonists on the relationships of inoculum density to infection incidence and disease severity in Fusarium crown rot of tomato. Phytopathology, 71(2), 167-170.

Mezzomo, Ricardo, Jéssica Mengue Rolim, Tales Poletto, Marília Boff De Oliveira, Marília Lazarotto, and Marlove Fátima Brião Muniz. 2018. "Mycelial Growth and Sporulation of Fusarium Spp. Pathogenic to Ilex Paraguariensis in Different Culture Media and under Exposure to Different Light Levels." Scientia Agraria 19 (1): 14-19. https://doi.org/10.5380/rsa.v19i1.55844.

Silva, J. L.; Teixeira, R. N. V. 2012. Esporulação e crescimento micelial de Fusarium solani em diferentes meios de cultura e regimes de luminosidade. RevistaAgro@mbiente On-line, v. 6, n. 1, p. 47-52.

Sreegayathri, E., G. Karthikeyan, L. Rajendran, A. Shanthi, and R. Ram Jegathesh. 2018. "Effect of Root Knot Nematode (Meloidogyne Incognita) Infestation on Severity of Wilt (Fusarium Solani) in Bitter Gourd and Its Management." Madras Agricultural Journal $105 \quad(10-12)$. https://doi.org/10.29321/maj.2018.00020 7.

Tamilselvi, N. A., L. Pugalendhi, and T. Raguchander. 2016. "Exploiting Cucurbitaceous Species as Rootstocks for Management of Fusarium Wilt (Fusarium Oxysporum) in Bitter Gourd." Australian Journal of Crop Science 10 (10): 1460-65. https://doi.org/10.21475/ajcs.2016.10.10. p7750.

\section{How to cite this article:}

Manohari, J., P. Latha, A. Kamalakannan, S. Selvakumar and Karthikeyan, M. 2020. Characterization of Fusarium solani (Mart.) Sacc. Causing Fusarium wilt of Bitter gourd in Coimbatore Region. Int.J.Curr.Microbiol.App.Sci. 9(06): 2336-2349. doi: https://doi.org/10.20546/ijcmas.2020.906.286 